\title{
Relationship between Insulin Secretion and Arterial Stiffness in Essential Hypertension
}

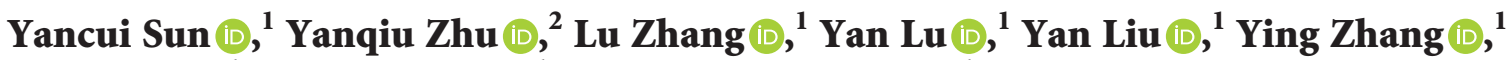 \\ Wei Song $\mathbb{D},{ }^{1}$ Yinong Jiang $\mathbb{D},{ }^{1}$ and Yunpeng Cheng $\mathbb{D}^{1}$ \\ ${ }^{1}$ Department of Cardiology, The First Affiliated Hospital of Dalian Medical University, Dalian, Liaoning, China \\ ${ }^{2}$ Department of Ultrasonography, The Affiliated Hospital of Dalian University, Dalian, Liaoning, China \\ Correspondence should be addressed to Yunpeng Cheng; yunpeng_cheng@hotmail.com
}

Received 17 September 2021; Accepted 8 December 2021; Published 24 December 2021

Academic Editor: Thereza Maria Magalhães Moreira

Copyright (C) 2021 Yancui Sun et al. This is an open access article distributed under the Creative Commons Attribution License, which permits unrestricted use, distribution, and reproduction in any medium, provided the original work is properly cited.

The study aims to explore the relationship between plasma insulin secretion and arterial stiffness in nondiabetic essential hypertensive patients. A total of 730 nondiabetic essential hypertensive patients registered between January 2016 and October 2020 were enrolled. A two-hour oral glucose tolerance test (OGTT) was performed to detect the levels of C-peptide and blood glucose at 0 hours and 2 hours, as well as the difference between C-peptide ( $\Delta$ C-peptide) and blood glucose ( $\Delta$ blood glucose) over the same period. Patients were divided into two groups: the normal glucose tolerance (NGT) group $(n=322)$ and the impaired glucose tolerance (IGT) group $(n=408)$. A multiple linear regression analysis was used to evaluate the association between brachial-ankle pulse wave velocity (baPWV) and the other factors. $0 \mathrm{~h} \mathrm{C}$-peptide, $2 \mathrm{~h}$ C-peptide, and $\Delta \mathrm{C}$-peptide were found to be higher in the IGT group. baPWV was positively linear correlated with $2 \mathrm{~h}$ C-peptide $(r=0.086, p=0.020)$ and $\Delta \mathrm{C}$-peptide $(r=0.115, p=0.002)$. baPWV remained independently associated with $0 \mathrm{~h}$ C-peptide, $2 \mathrm{~h} \mathrm{C}$-peptide, and $\Delta \mathrm{C}$-peptide, after adjusting by age, gender, smoking, body mass index (BMI), high-density lipoprotein (HDL), cholesterol, systolic blood pressure (SBP), and triglycerides (TG). Our data shows that higher endogenous insulin secretion might play an important role in the progression of arterial stiffness in nondiabetic essential hypertensive patients.

\section{Introduction}

Hypertension is a leading risk factor of cardiovascular diseases. Many studies have revealed that hypertension, in the absence of obesity or diabetes mellitus, is associated with insulin resistance [1-3]. When insulin resistance appears, insulin levels are elevated, and the resulting increase can be reflected by the detection of insulin content. C-peptide is cosecreted with insulin on an equimolar basis; this is more stable, has fewer influencing factors, and can better reflect the secretion ability of overall and residual islet $\beta$-cell [4-6]. In addition, C-peptide may also be a predictive marker of the severity of cardiovascular disease [7] and mortality [8]; the research suggests that $\mathrm{C}$-peptide is a bioactive peptide with other potential physiological functions. Meanwhile, the detection of C-peptide is simple and convenient.
Arterial stiffness is accompanied by an increase of pulse wave velocity, which is an important predictor of cardiovascular disease [9-12]. Several factors are believed to be responsible for arterial stiffness, including age, hypertension, and hyperglycemia. Studies have found that insulin resistance is significantly associated with arterial stiffness $[13,14]$. Euglycemic hyperinsulinemic clamp studies have revealed a relationship between insulin resistance and arterial stiffness in both young, healthy women and diabetic adults [15-17]. A few studies examining the existence of this relationship were conducted for individuals without diabetes. Additionally, studies involving the association between insulin secretion and arterial stiffness are limited.

Therefore, this study aims to determine the association between insulin secretion (assessed by C-peptide) and arterial stiffness (assessed by brachial-ankle pulse wave 
velocity (baPWV)) in the Chinese essential hypertensive population without diabetes.

\section{Methods}

2.1. Study Subjects. All subjects in this study were examined within the period of January 2016 to October 2020 at the First Affiliated Hospital of Dalian Medical University. The inclusion criteria are as follows: (1) hypertensive patients classified according to the 2018 European Society of Cardiology and the European Society of Hypertension (ESC/ ESH) guidelines office [18], with systolic blood pressure (SBP) at a value $\geq 140 \mathrm{mmHg}$ or diastolic blood pressure (DBP) at a value $\geq 90 \mathrm{mmHg}$, using antihypertensive drugs, and (2) $\geq 18$ years old. The exclusion criteria are as follows: (1) diagnosis of secondary hypertension $(n=106)$, (2) diagnosis of diabetes mellitus $(n=493)$, (3) severe arrhythmia $(n=34)$, (4) moderate to severe kidney dysfunction (estimated glomerular filtration rate $(\mathrm{eGFR}) \leq 60 \mathrm{ml} \mathrm{min} \mathrm{m}^{-1}$ $\left.1.73 \mathrm{~m}^{2}\right)(n=122)$, (5) left ventricular systolic dysfunction (LVEF <50\%) $(n=58)$, (6) severe valvular heart disease $(n=26),(7)$ peripheral vascular diseases (PAD) $(n=62),(8)$ myocardial infarction or unstable angina pectoris $(n=156)$, and (9) those subjects missing dates of baPWV or C-peptide $(n=213)$. Finally, the present study contained a total of 730 subjects. The research was conducted in accordance with the Declaration of Helsinki and was approved by the institutional review board of the First Affiliated Hospital of Dalian Medical University. The informed consent provision was waived, and all procedures listed here were carried out in compliance with the approved guidelines.

2.2. Clinical Measurements and Definition of Explanatory Variables. Demographic and clinical characteristics include age, gender, height, weight, whether the subject is a current smoker, cardiovascular disease, use of oral antihypertensive drugs, and use of statins. Participants were deemed current smokers if they reported that they were currently smoking or had registered smoking at least 100 cigarettes during their lifetime $[19,20]$. Body mass index (BMI) was calculated according to the following formula: weight $(\mathrm{kg}) /$ height $(\mathrm{m})^{2}$. A sample of fasting blood from the brachial vein was collected, and the biochemical parameters included total cholesterol (TC), triglycerides (TG), low-density lipoprotein (LDL) cholesterol, and high-density lipoprotein (HDL) cholesterol. The sample collections were performed at the First Affiliated Hospital of Dalian Medical University using the standard protocols. eGFR was calculated according to the equation suggested by the Chronic Kidney Disease Epidemiology (CKD-EPI) Collaboration [21]. PAD was defined as ankle-brachial index $(\mathrm{ABI})<0.9$, stent implantation, or according to past medical records.

2.3. Oral Glucose Tolerance Test (OGTT). Blood samples for glucose and $\mathrm{C}$-peptide were obtained at 0 hours and 2 hours after a 75-g oral glucose load. Serum C-peptide levels were measured by chemiluminescence immunoassay, while serum glucose concentrations were assayed using a glucose oxidase assay. Hemoglobin A1c (HbA1c) analysis was performed on whole blood using high-performance liquid chromatography. The diagnosis of diabetes and prediabetes was defined based on the World Health Organization's 1999 criteria after the OGTT [22]. Participants were divided into two groups: the normal glucose tolerance (NGT) group and the impaired glucose tolerance (IGT) group. NGT was diagnosed if the fasting plasma glucose level was $<6.1 \mathrm{mmol} / \mathrm{L}$ and the $2 \mathrm{~h}$ value was $<7.8 \mathrm{mmol} / \mathrm{L}$. IGT was diagnosed if the fasting plasma glucose level was $<7.0 \mathrm{mmol} / \mathrm{L}$ but the $2 \mathrm{~h}$ value was $7.8-11.1 \mathrm{mmol} / \mathrm{L}$, or if fasting glucose was $6.1-7.0 \mathrm{mmol} / \mathrm{L}$ but the $2 \mathrm{~h}$ glucose value was $<7.8 \mathrm{mmol} / \mathrm{L}$.

The plasma glucose difference ( $\Delta$ plasma glucose) was defined as $2 \mathrm{~h}$ plasma glucose $-0 \mathrm{~h}$ plasma glucose, whereas the C-peptide difference ( $\Delta \mathrm{C}$-peptide) was defined as $2 \mathrm{~h} \mathrm{C}$ peptide - 0 h C-peptide.

2.4. Ambulatory Blood Pressure. Blood pressure was measured on an ambulatory basis using an automatic 24-hour monitor (model 90207; Spacelabs, Issaquah, WA). Measurements were taken every 30 minutes during the daytime period (0700-2300 h) and every hour during the night period (2300-0700 h). These measurements were averaged to produce the mean $24 \mathrm{~h}$ systolic blood pressure (SBP), diastolic blood pressure (DBP), and heart rate (HR).

2.5. Measurement of baPWV. baPWV measurements recorded in the supine position were obtained using a volume-plethysmography device (VP-1000, OMRON, Dalian, China), which measures both brachial and posterior tibial artery pressure waveforms using an oscillometric method with cuffs placed around the participant's arms and ankles. baPWV was calculated automatically via time-phase analysis, and the distance between the upper arm and ankle was estimated based on the participant's height. baPWV was obtained from right and left measurements, and the higher of the two readings was used for analysis [23].

2.6. Statistical Analysis. All statistical analyses were conducted using SPSS version 26.0. Normally distributed continuous variables were recorded as mean \pm standard deviation. Nonnormal distribution parameters were transformed or presented as the median (25th -75 th percentile). Categorical data were presented as number and percentages $(n, \%)$. Differences between groups were compared by using Student's $t$-test, the Chi-squared test, and Mann-Whitney's $U$ test. Spearman correlation or Kendall correlation analysis was used to explore the association between baPWV and other factors, while multiple linear regression analysis was used to evaluate this association. All statistical analyses were two-sided, and a $P$ value of less than 0.05 was considered statistically significant.

\section{Results}

3.1. Baseline Characteristics of the Participants. During the study period, 730 subjects had sufficient data to evaluate the baPWV and C-peptide. The baseline characteristics of 
participants are shown in Table 1. The mean levels of BMI, $0 \mathrm{~h}$ plasma glucose $(0 \mathrm{~h} \mathrm{PG}), 2 \mathrm{~h}$ plasma glucose $(2 \mathrm{~h} \mathrm{PG}), \Delta$ plasma glucose, $0 \mathrm{~h} \mathrm{C}$-peptide, $2 \mathrm{~h} \mathrm{C}$-peptide, $\Delta \mathrm{C}$-peptide, $\mathrm{HbAlc}$, and TG in the IGT group were found to be significantly higher than those in the NGT group (all $P<0.05$ ). Meanwhile, the mean level of HDL-C was lower in the IGT group $(P<0.001)$. Other factors include age, female, current smoker, eGFR, TC, LDL-cholesterol, HR, SBP, DBP, baPWV, stable angina, use of antihypertension drugs, and use of statin were not statistically significant in two groups.

3.2. Univariate Correlation with baPWV. As shown in Table 2, correlation analysis indicated that baPWV was positively correlated with age $(r=0.407, \quad P<0.001), 2 \mathrm{~h}$ C-peptide $(r=0.086, P=0.020), \Delta$ C-peptide $(r=0.115, P=0.002)$, HDL-C $(r=0.158, P<0.001)$, HbAlc $(r=0.077, P=0.037)$, $\operatorname{SBP}(r=0.211, P<0.001)$, stable angina $(r=0.081, P=0.029)$, calcium channel blocker (CCB) $(r=0.160, P<0.001)$, diuretics $(r=0.167, P<0.001)$, and statin $(r=0.178, P<0.001)$. The other factors, namely, female $(r=-0.181, P<0.001)$, current smoking $(r=-0.151, P<0.001)$, BMI $(r=-0.235, P<0.001)$, eGFR $(r=-0.125, P=0.001)$, and TG $(r=-0.100, P=0.007)$ were found to be negatively correlated with baPWV.

\subsection{Association between baPWV and the C-Peptide Correlation} Index. The results of the multiple linear regression models used to determine the degree to which each of the different covariates impacted baPWV are presented in Table 3 as $\beta$ and $95 \%$ confidence intervals (CIs). Model 1 was not adjusted, model 2 was adjusted for age and sex, and model 3 was adjusted for age, sex, current smoking, BMI, HDL-C, mean 24h SBP, and TG. After controlling for confounding factors, it was found that $0 \mathrm{~h}$ C-peptide $(\beta=0.091$, $P=0.012), 2$ hC-peptide $(\beta=0.089, P=0.006)$, and $\Delta$ C-peptide $(\beta=0.078, P=0.014)$ were positively associated with baPWV.

\section{Discussion}

Hypertension is associated with insulin resistance (IR), metabolic syndrome (MS), and arterial stiffness [24]. Some studies show there is a correlation between insulin resistance and arterial stiffness [15-17]; however, there is a lack of research on the relationship between insulin secretion and arterial stiffness. The research paper aims to explore the relationship between insulin secretion and arterial stiffness.

Although C-peptide is not a direct measure of $\beta$ cell mass, its plasma levels provide a fairly accurate estimate of endogenous insulin secretion. Some studies show that assessing the $\beta$-cell function as a measurement of C-peptide after stimulation may be advantageous [25]. $2 \mathrm{~h} \mathrm{C}$-peptide represents the insulin content after 2 hours of glucose tolerance, while $\Delta$ C-peptide reflects the ability of pancreatic islet $\beta$ cells to secrete insulin after glucose tolerance. The main finding of this study is that $0 \mathrm{~h} \mathrm{C}$-peptide $(\beta=0.091, P=0.012), 2 \mathrm{~h} \mathrm{C}$ peptide $(\beta=0.089, P=0.006)$, and $\Delta \mathrm{C}$-peptide $(\beta=0.078$, $P=0.014)$ are associated with baPWV, and this association appeared to be independent of glucose tolerance status in hypertensive nondiabetic subjects. Furthermore, the association remained positive after adjusting for age, sex, BMI, and other confounding factors. Higher $\Delta \mathrm{C}$-peptide may represent higher endogenous insulin secretion and may be a compensatory factor of insulin resistance due to higher baPWV, which implies that higher $\Delta \mathrm{C}$-peptide plays an important role in the progress of arterial stiffness.

Previous studies have shown that age, gender, and BMI are associated with increased arterial stiffness [26, 27]. The present study confirms the association between age, gender, BMI, and arterial stiffness in this population. The study also found, through the multiple regression models, that the relation between insulin secretion and arterial stiffness was independent of age, gender, and BMI. These results suggest that the relationship between age, gender, or BMI and arterial stiffness may be mediated in part through the increase of insulin secretion.

The mechanism underlying the relationship between insulin secretion and arterial stiffness is unknown, and this crosssectional study cannot identify the causative factor. The hyperinsulinemic response by the $\beta$ cells is multifactorial, including adaptive changes in $\beta$ cell mass and function. Related literature has shown that the increase of insulin secretion is reflected by the mass of islet cells rather than the secretion capacity of pancreatic $\beta$ cells [28-30]. The present study finds that, under a long-term high-pressure environment, $\beta$ cells initially mount a compensatory response to ramp up $\beta$ cell functional capacity and insulin secretion to meet the elevated pressure demand. Other studies have found that insulin resistance leads to arterial stiffness [13, 14, 31]. The outcome of the abovementioned mechanisms is increased insulin secretion. Abnormal secretion of insulin can enhance the activity of the renin-angiotensin-aldosterone system (RAAS) and the sympathetic nervous system. The results of these changes involve the increase in the secretion of angiotensin, aldosterone, catecholamine, and endothelin 1 , as well as the promotion of the contraction and remodeling of blood vessels, leading to arterial stiffness [32]. In addition, excessive RAAS activation increases the expression of distal renal tubular sodium channels, enhances the activity of vascular endothelial sodium channels, and promotes fibrosis, remodeling, diastolic dysfunction of arterial and salt sensitivity in blood pressure regulation. Finally, these changes aggravate blood pressure and the progression of arterial stiffness [33].

This study did not identify an increase in arterial stiffness among individuals with IGT. This finding was consistent with previous studies that had failed to identify a significant increase in central arterial stiffness measurements among those with IGT compared with those with NGT [31]. A previous study found that hyperglycemia is associated with significant arterial stiffening and that postchallenge glucose and the homeostasis model assessment of insulin resistance (HOMA-IR) index are the most powerful metabolic predictors of arterial stiffness [34]. This research, however, finds no association between arterial stiffness and glucose measurements including $0 \mathrm{~h} \mathrm{PG}, 2 \mathrm{~h}$ PG, and $\Delta$ plasma glucose level. The mechanism underlying the relationship is unknown, and this cross-sectional study cannot identify the 
TABLE 1: Baseline characteristics of the participants.

\begin{tabular}{|c|c|c|c|c|}
\hline Variables & NGT $(n=322)$ & IGT $(n=408)$ & Total $(n=730)$ & $P$ value \\
\hline Age (years) & $55.2 \pm 13.1$ & $54.5 \pm 13.1$ & $54.8 \pm 13.1$ & 0.448 \\
\hline Female, $n(\%)$ & $147(45.7)$ & $182(44.6)$ & $329(45.1)$ & 0.778 \\
\hline Current smoking, $n(\%)$ & $74(23)$ & $111(27.2)$ & $185(25.3)$ & 0.396 \\
\hline BMI $\left(\mathrm{kg} / \mathrm{m}^{2}\right)$ & $26.6 \pm 3.9$ & $27.7 \pm 4.0$ & $27.2 \pm 4.0$ & $<0.001$ \\
\hline $\mathrm{eGFR}\left(\mathrm{ml} \mathrm{min}{ }^{-1} 1.73 \mathrm{~m}^{2}\right)$ & $104.7 \pm 20.5$ & $106.9 \pm 22.6$ & $105.9 \pm 21.7$ & 0.174 \\
\hline 0 h PG $(\mathrm{mmol} / \mathrm{L})$ & $4.7 \pm 0.5$ & $5.1 \pm 0.6$ & $4.9 \pm 0.6$ & $<0.001$ \\
\hline $2 \mathrm{~h} \mathrm{PG}(\mathrm{mmol} / \mathrm{L})$ & $6.7 \pm 0.6$ & $9.3 \pm 0.9$ & $8.2 \pm 1.5$ & $<0.001$ \\
\hline$\Delta \mathrm{PG}(\mathrm{mmol} / \mathrm{L})$ & $2.0 \pm 0.6$ & $4.2 \pm 1.0$ & $3.2 \pm 1.4$ & $<0.001$ \\
\hline 0 h C-peptide (ng/ml) & $2.0(1.7-2.6)$ & $2.3(1.8-2.9)$ & $2.2(1.8-2.7)$ & $<0.001$ \\
\hline 2 h C-peptide (ng/ml) & $8.0(6.3-9.9)$ & $10.2(8.5-12.7)$ & $9.2(7.3-11.5)$ & $<0.001$ \\
\hline$\Delta \mathrm{C}$-peptide $(\mathrm{ng} / \mathrm{ml})$ & $5.9(4.5-7.6)$ & $7.7(6.4-10.2)$ & $7.1(5.4-8.9)$ & $<0.001$ \\
\hline HbAlc (\%) & $5.9 \pm 0.3$ & $6.0 \pm 0.4$ & $6.0 \pm 0.4$ & $<0.001$ \\
\hline $\mathrm{TC}(\mathrm{mmol} / \mathrm{L})$ & $5.1(4.4-5.8)$ & $5.0(4.3-5.7)$ & $5.0(4.3-5.7)$ & 0.420 \\
\hline $\mathrm{TG}(\mathrm{mmol} / \mathrm{L})$ & $1.4(1.0-2.0)$ & $1.5(1.1-2.1)$ & $1.5(1.1-2.1)$ & 0.012 \\
\hline $\mathrm{HDL}-\mathrm{C}(\mathrm{mmol} / \mathrm{L})$ & $1.3 \pm 0.4$ & $1.2 \pm 0.3$ & $1.2 \pm 0.3$ & $<0.001$ \\
\hline LDL-C (mmol/L) & $2.8(2.4-3.3)$ & $2.8(2.3-3.3)$ & $2.8(2.3-3.3)$ & 0.659 \\
\hline Mean $24 \mathrm{~h}$ heart rate (beats/min) & $69.9 \pm 8.8$ & $70.9 \pm 9.3$ & $70.5 \pm 9.1$ & 0.134 \\
\hline Mean 24h SBP (mmHg) & $169.8 \pm 20.2$ & $172.0 \pm 21.4$ & $171.0 \pm 20.9$ & 0.156 \\
\hline Mean 24h DBP (mmHg) & $107.9 \pm 17.0$ & $108.5 \pm 16.2$ & $108.2 \pm 16.5$ & 0.601 \\
\hline $\mathrm{baPWV}(\mathrm{cm} / \mathrm{s})$ & $1555.3(1398.8-1749.0)$ & $1556.0(1371.0-1780.3)$ & $1555.8(1385.8-1762.6)$ & 0.884 \\
\hline Stable angina, $n(\%)$ & $47(14.6)$ & $61(15)$ & $108(14.8)$ & 0.893 \\
\hline Calcium channel blocker, $n(\%)$ & $226(70.2)$ & $302(74.0)$ & $528(72.3)$ & 0.250 \\
\hline ACEI, $n(\%)$ & $7(2.2)$ & $13(3.2)$ & $20(2.7)$ & 0.405 \\
\hline $\mathrm{ARB}, n(\%)$ & $218(67.7)$ & $271(66.4)$ & $489(67)$ & 0.715 \\
\hline$\beta$-Blocker, $n(\%)$ & $132(41.0)$ & $170(41.7)$ & $302(41.4)$ & 0.855 \\
\hline Diuretic, $n(\%)$ & $69(21.4)$ & $83(20.3)$ & $152(20.8)$ & 0.720 \\
\hline$\alpha$-Blocker, $n(\%)$ & $25(7.8)$ & $29(7.1)$ & $54(7.4)$ & 0.737 \\
\hline Statins use, $n(\%)$ & $199(61.8)$ & $242(59.3)$ & $441(60.4)$ & 0.495 \\
\hline
\end{tabular}

Values are mean $\pm S D$, median (25th and 75th percentiles), or number of patients (percentage of column total). BMI, body mass index; eGFR, estimated glomerular filtration rate; PG, plasma glucose; HbAlc, hemoglobin A1c; TC, total cholesterol; TG, triglycerides; HDL-C, high-density lipoprotein cholesterol; LDL-C, low-density lipoprotein; SBP, systolic blood pressure; DBP, diastolic blood pressure; baPWV, brachial-ankle pulse wave velocity; ACEI, angiotensinconverting enzyme inhibitor; ARB, angiotensin-converting enzyme receptor blocker.

TABLE 2: Univariate correlation with baPWV.

\begin{tabular}{|c|c|c|}
\hline Variables & $\mathrm{r}$ & $P$ value \\
\hline Age (years) & 0.407 & $<0.001$ \\
\hline Female, $n(\%)$ & -0.181 & $<0.001$ \\
\hline Current smoking, $n(\%)$ & -0.151 & $<0.001$ \\
\hline BMI $\left(\mathrm{kg} / \mathrm{m}^{2}\right)$ & -0.235 & $<0.001$ \\
\hline $\mathrm{eGFR}\left(\mathrm{ml} \mathrm{min}{ }^{-1} 1.73 \mathrm{~m}^{2}\right)$ & -0.125 & 0.001 \\
\hline 0 h PG $(\mathrm{mmol} / \mathrm{L})$ & 0.052 & 0.158 \\
\hline $2 \mathrm{~h}$ PG $(\mathrm{mmol} / \mathrm{L})$ & 0.001 & 0.988 \\
\hline$\Delta \mathrm{PG}(\mathrm{mmol} / \mathrm{L})$ & -0.016 & 0.675 \\
\hline 0 h C-peptide (ng/ml) & -0.043 & 0.249 \\
\hline 2 h C-peptide (ng/ml) & 0.086 & 0.020 \\
\hline$\Delta$ C-peptide $(\mathrm{ng} / \mathrm{ml})$ & 0.115 & 0.002 \\
\hline HbAlc (\%) & 0.077 & 0.037 \\
\hline $\mathrm{TC}(\mathrm{mmol} / \mathrm{L})$ & 0.065 & 0.079 \\
\hline $\mathrm{TG}(\mathrm{mmol} / \mathrm{L})$ & -0.100 & 0.007 \\
\hline HDL-C (mmol/L) & 0.158 & $<0.001$ \\
\hline LDL-C (mmol/L) & 0.029 & 0.435 \\
\hline Heart rate (beats/min) & 0.025 & 0.497 \\
\hline Mean 24 h SBP (mmHg) & 0.211 & $<0.001$ \\
\hline Mean 24 h DBP (mm Hg) & -0.015 & 0.678 \\
\hline Stable angina, $n(\%)$ & 0.081 & 0.029 \\
\hline Calcium channel blocker, $n(\%)$ & 0.160 & $<0.001$ \\
\hline ACEI, $n(\%)$ & 0.009 & 0.805 \\
\hline $\mathrm{ARB}, n(\%)$ & 0.062 & 0.095 \\
\hline$\beta$-Blocker, $n(\%)$ & 0.032 & 0.386 \\
\hline
\end{tabular}


TABLE 2: Continued.

\begin{tabular}{lcc}
\hline Variables & $\mathrm{r}$ & $P$ value \\
\hline Diuretic, $n(\%)$ & 0.167 & $<0.001$ \\
$\alpha$-Blocker, $n(\%)$ & 0.012 & 0.751 \\
Statins use, $n(\%)$ & 0.178 & $<0.001$ \\
\hline
\end{tabular}

baPWV, brachial-ankle pulse wave velocity; BMI, body mass index; eGFR, estimated glomerular filtration rate; PG, plasma glucose; HbAlc, hemoglobin A1c; TC, total cholesterol; TG, triglycerides; HDL-C, high-density lipoprotein cholesterol; LDL-C, low-density lipoprotein; SBP, systolic blood pressure; DBP, diastolic blood pressure; ACEI, angiotensin-converting enzyme inhibitor; ARB, angiotensin-converting enzyme receptor blocker.

TABLE 3: Multiple linear regression analysis showing variables independently associated with baPWV.

\begin{tabular}{lccccccccc}
\hline Variables & \multicolumn{3}{c}{ Model 1 } & \multicolumn{3}{c}{ Model 2 } & & \multicolumn{2}{c}{ Model 3 } \\
& $\beta$ & $95 \%$ CI & $P$ value & $\beta$ & $95 \%$ CI & $P$ value & $\beta$ & $95 \%$ CI & $P$ value \\
\hline 0 h PG $(\mathrm{mmol} / \mathrm{L})$ & 0.055 & $-9.568--67.659$ & 0.14 & 0.021 & $-24.495--47.317$ & 0.533 & 0.037 & $-13.541--53.059$ & 0.244 \\
2 h PG $(\mathrm{mmol} / \mathrm{L})$ & 0.032 & $-8.266--21.300$ & 0.387 & 0.039 & $-5.763--21.586$ & 0.256 & 0.035 & $-5.553--19.844$ & 0.270 \\
$\Delta$ PG $(\mathrm{mmol} / \mathrm{L})$ & 0.012 & $-13.292--18.549$ & 0.746 & 0.033 & $-7.477--22.005$ & 0.334 & 0.022 & $-8.710--18.532$ & 0.479 \\
0 h C-peptide $(\mathrm{ng} / \mathrm{ml})$ & -0.062 & $-44.579--3.651$ & 0.096 & 0.037 & $-10.729--35.498$ & 0.293 & 0.091 & $6.782--53.487$ & 0.012 \\
2 h C-peptide (ng/ml) & 0.077 & $0.389--12.906$ & 0.037 & 0.077 & $0.865--12.442$ & 0.024 & 0.089 & $2.237--13.094$ & 0.006 \\
$\Delta$ C-peptide (ng/ml) & 0.106 & $3.323--17.558$ & 0.004 & 0.078 & $1.069--14.318$ & 0.023 & 0.078 & $1.534--13.777$ & 0.014 \\
\hline
\end{tabular}

Model 1: not adjusted. Model 2: adjusted for age and sex. Model 3: adjusted for age, sex, current smoking, BMI, HDL-C, mean 24 h SBP, and TG. CI, confidence interval; baPWV, brachial-ankle pulse wave velocity; PG, plasma glucose; BMI, body mass index; HDL-C, high-density lipoprotein cholesterol; SBP, systolic blood pressure; TG, triglycerides.

causative factor; however, the duration of diabetes and the level of blood glucose may be explored in future studies.

It is important to note several limitations in this study design. First, the cross-sectional design restricts the causal relationship between the baPWV and the C-peptide. Second, only one center is included in this study. Our study sample lacks national or regional representation; this limits the ability to generalize the results at an international level. Thus, to improve the consistency of these findings, the study may require replication in other parts of the country or in other regions of the world. Finally, the measurements taken for glucose exposure (fasting, $2 \mathrm{~h}$ OGTT, $\Delta$ plasma glucose level, and glucose tolerance status) may not capture glucose exposure over time. To that end, future studies addressing this association should be designed to incorporate measures that may better reflect glucose exposure over time.

In conclusion, the results emerging from our study strengthen the available evidence on the importance of the secretion level of islet $\beta$ cells as a cause and predictor of arterial stiffness in nondiabetic essential hypertensive patients. Furthermore, our data appears highly clinically relevant since knowledge of these pathophysiological mechanisms may allow early interventions on vascular damage, which in turn may prevent clinical events.

\section{Data Availability}

The datasets used and analyzed during the current study are available from the corresponding author on reasonable request.

\section{Conflicts of Interest}

The authors declare that they have no conflicts of interest.

\section{Authors' Contributions}

Yancui Sun and Yanqiu Zhu contributed equally to this work.

\section{References}

[1] E. Ferrannini, G. Buzzigoli, R. Bonadonna et al., "Insulin resistance in essential hypertension," New England Journal of Medicine, vol. 317, no. 6, pp. 350-357, 1987.

[2] G. M. Reaven, "Relationship between insulin resistance and hypertension," Diabetes Care, vol. 14, no. 4, pp. 33-38, 1991.

[3] M. Laakso, H. Sarlund, and L. Mykkänen, "Essential hypertension and insulin resistance in non-insulin-dependent diabetes," European Journal of Clinical Investigation, vol. 19, no. 6, pp. 518-526, 1989.

[4] K. Polonsky, B. Frank, W. Pugh et al., "The limitations to and valid use of C-peptide as a marker of the secretion of insulin," Diabetes, vol. 35, no. 4, pp. 379-386, 1986.

[5] K. S. Polonsky, B. D. Given, L. Hirsch et al., "Quantitative study of insulin secretion and clearance in normal and obese subjects," Journal of Clinical Investigation, vol. 81, no. 2, pp. $435-441,1988$.

[6] C. Ma, Y. Liu, S. He et al., "C-peptide: a mediator of the association between serum uric acid to creatinine ratio and non-alcoholic fatty liver disease in a Chinese population with normal serum uric acid levels," Frontiers in Endocrinology, vol. 11, p. 600472, 2020.

[7] B. Harnishsingh and B. Rama, "Is C-peptide a predictor of severity of coronary artery disease in metabolic syndrome? An 
observational study," Indian Heart Journal, vol. 70, no. 3, pp. S105-S109, 2018.

[8] J.-y. Min and K.-b. Min, "Serum C-peptide levels and risk of death among adults without diabetes mellitus," Canadian Medical Association Journal, vol. 185, no. 9, pp. E402-E408, 2013.

[9] Y. Ni, H. Wang, D. Hu, and W. Zhang, "The relationship between pulse wave velocity and pulse pressure in Chinese patients with essential hypertension," Hypertension Research, vol. 26, no. 11, pp. 871-874, 2003.

[10] G. Schillaci, F. Battista, L. Settimi, F. Anastasio, and G. Pucci, "Cardio-ankle vascular index and subclinical heart disease," Hypertension Research, vol. 38, no. 1, pp. 68-73, 2015.

[11] A. László, G. Reusz, and J. Nemcsik, "Ambulatory arterial stiffness in chronic kidney disease: a methodological review," Hypertension Research, vol. 39, no. 4, pp. 192-198, 2016.

[12] D. Botha, Y. Breet, and A. E. Schutte, "Comparing the associations of clinic vs. ambulatory blood pressure with subclinical organ damage in young healthy adults: the AfricanPREDICT study," Hypertension Research, vol. 44, no. 7, pp. 840-849, 2021.

[13] M. A. Hill, Y. Yang, L. Zhang et al., "Insulin resistance, cardiovascular stiffening and cardiovascular disease," Metabolism, vol. 119, p. 154766, 2021.

[14] V. Cassano, D Crescibene, M. L Hribal et al., "Uric acid and vascular damage in essential hypertension: role of insulin resistance," Nutrients, vol. 12, no. 9, 2020.

[15] E. J. Giltay, J. Lambert, J. M. H. Elbers, L. J. G. Gooren, H. Asscheman, and C. D. A. Stehouwer, "Arterial compliance and distensibility are modulated by body composition in both men and women but by insulin sensitivity only in women," Diabetologia, vol. 42, no. 2, pp. 214-221, 1999.

[16] R. A. J. M. van Dijk, S. J. L. Bakker, P. G. Scheffer, R. J. Heine, and C. D. A. Stehouwer, "Associations of metabolic variables with arterial stiffness in type 2 diabetes mellitus: focus on insulin sensitivity and postprandial triglyceridaemia," European Journal of Clinical Investigation, vol. 33, no. 4, pp. 307-315, 2003.

[17] M. Emoto, Y. Nishizawa, T. Kawagishi et al., "Stiffness indexes of the common carotid and femoral arteries are associated with insulin resistance in NIDDM," Diabetes Care, vol. 21, no. 7, pp. 1178-1182, 1998.

[18] B. Williams, "ESC/ESH Guidelines for the management of arterial hypertension," European Heart Journal, vol. 39, no. 33, pp. 3021-3104, 2018.

[19] D. E. Bild, "Multi-ethnic study of atherosclerosis: objectives and design," American Journal of Epidemiology, vol. 156, no. 9, pp. 871-881, 2002.

[20] E. S. Ford, W. H. Giles, and A. H. Mokdad, "The distribution of 10-Year risk for coronary heart disease among U.S. adults," Journal of the American College of Cardiology, vol. 43, no. 10, pp. 1791-1796, 2004.

[21] A. S. Levey, L. A. Stevens, C. H. Schmid et al., "A new equation to estimate glomerular filtration rate," Annals of Internal Medicine, vol. 150, no. 9, pp. 604-612, 2009.

[22] K. G. M. M. Alberti and P. Z. Zimmet, "Definition, diagnosis and classification of diabetes mellitus and its complications. Part 1: diagnosis and classification of diabetes mellitus. Provisional report of a WHO Consultation," Diabetic Medicine, vol. 15, no. 7, pp. 539-553, 1998.

[23] J.-Y. Lee, S. Ryu, S. H. Lee et al., “Association between brachial-ankle pulse wave velocity and progression of coronary artery calcium: a prospective cohort study," Cardiovascular Diabetology, vol. 14, no. 1, p. 147, 2015.
[24] M. Cecelja and P. Chowienczyk, "Dissociation of aortic pulse wave velocity with risk factors for cardiovascular disease other than hypertension," Hypertension, vol. 54, no. 6, pp. 1328-1336, 2009.

[25] S. D. Hsieh, Y. Iwamoto, A. Matsuda, and T. Kuzuya, "Pancreatic B-cell function in non-insulin-dependent diabetes mellitus during successive periods of sulfonylurea and insulin treatement: serum C-peptide response to glucagon and urine C-peptide excretion," Endocrinologia Japonica, vol. 34, no. 4, pp. 561-567, 1987.

[26] A. Vallée, V. Olié, H. é. l. Lelong, S. Kretz, M. E. Safar, and J. Blacher, "Relationship between BMI and aortic stiffness: influence of anthropometric indices in hypertensive men and women," Journal of Hypertension, vol. 38, no. 2, pp. 249-256, 2020.

[27] L. Woznicka-Leskiewicz, A. Posadzy-Malaczynska, and J. Marcinkowska, "Gender, subclinical organ damage and cardiovascular risk stratification in hypertensive patients," Current Medical Research and Opinion, vol. 35, no. 2, pp. 367-374, 2019.

[28] L. Fransson, S. Franzén, V. Rosengren, P. Wolbert, $\AA$ A S Söholm, and H. Ortsäter, " $\beta$-cell adaptation in a mouse model of glucocorticoid-induced metabolic syndrome," Journal of Endocrinology, vol. 219, no. 3, pp. 231-241, 2013.

[29] Y. Saisho, A. E. Butler, E. Manesso, D. Elashoff, R. A. Rizza, and P. C. Butler, "-cell mass and turnover in humans: effects of obesity and aging," Diabetes Care, vol. 36, no. 1, pp. 111-117, 2013.

[30] T. Mezza, G. Muscogiuri, G. P. Sorice et al., "Insulin resistance alters islet morphology in nondiabetic humans," Diabetes, vol. 63, no. 3, pp. 994-1007, 2014.

[31] D. M. Sengstock, P. V. Vaitkevicius, and M. A. Supiano, "Arterial stiffness is related to insulin resistance in nondiabetic hypertensive older adults," Journal of Clinical Endocrinology \& Metabolism, vol. 90, no. 5, pp. 2823-2827, 2005.

[32] R. Muniyappa, H. Chen, M. Montagnani, A. Sherman, and M. J. Quon, "Endothelial dysfunction due to selective insulin resistance in vascular endothelium: insights from mechanistic modeling," American Journal of Physiology. Endocrinology and Metabolism, vol. 319, no. 3, pp. E629-E646, 2020.

[33] G. Jia, J. Habibi, A. R. Aroor et al., "Endothelial mineralocorticoid receptor mediates diet-induced aortic stiffness in females," Circulation Research, vol. 118, no. 6, pp. 935-943, 2016.

[34] D. R. Webb, K. Khunti, R. Silverman et al., "Impact of metabolic indices on central artery stiffness: independent association of insulin resistance and glucose with aortic pulse wave velocity," Diabetologia, vol. 53, no. 6, pp. 1190-1198, 2010. 